\title{
Congestion Control for Wireless Sensor Network Based on Generalized Minimum Variance Controller
}

\author{
Xinhao Yang \\ Department of Mechanical and Electric Engineering \\ Soochow University \\ Suzhou, China \\ e-mail: yangxinhao@163.com
}

\section{$\mathrm{Ze} \mathrm{Li}$}

College of electronic and information engineering

Suzhou University of Science and Technology

Suzhou, China

e-mail: lizeing@163.com

\author{
Juncheng Jia \\ School of Computer Science and Technology \\ Soochow University \\ Suzhou, China \\ e-mail: jiajuncheng@suda.edu.cn \\ Shukui Zhang \\ School of Computer Science and Technology \\ Soochow University \\ Suzhou, China \\ e-mail: zhangshukui@suda.edu.cn
}

\begin{abstract}
In order to improve the quality of service and the energy efficiency, congestion control has exposed as an essential factor in the wireless sensor network. Congestion control for wireless sensor network based on generalized minimum variance controller is proposed in the paper, for which node-congestion and link-congestion are considered simultaneously. The discrete transfer functionfor the nodecongestion and the link-congestion, as the wireless sensor network model, is presented based on the fluid-flow theory, small signal linearization and the bilinear Z-transform. Due to the time-variable network parameter and topology, generalized minimum variance controller is introduced to the congestion control in wireless sensro network, of which the necessary and sufficient condition for the stability is also provided. The congestion control algorithm based on generalized minimum variance controller can reduce queue time and alleviate congestion. Ns-2 simulation results indicate that the proposed algorithm restrains the congestion in variable network condition and maintains a high throughput together with a low packet drop ratio for the whole network.
\end{abstract}

Keywords- congestion control; wireless sensor network; generalized minimum variance; Ns-2 simulantion; quality of service

\section{INTRODUCTION}

Over the last decades, there have been widely researches in the area of the wireless sensor networks(WSNs) [1]. Normally, the sensor nodes will be deployed in the remote area, such as the island in the sea and the satellite in the outerspace, in which case recharging is not feasible. Thus, the main focus for WSNs is on the low energy use. Congestion may result in wasted resources due to lost or dropped packets, and even possible congestion collapse. So congestion control is considered as an essential factor in WSNs.

In order to improve the quality of service and the energy efficiency, several congestion control methods are researched for sensor network applications. As the queue length in buffer suggesting the current network condition, IFRC [2] uses queue sizes to detect the congestion, and further shares the congestion state through overhearing. Rate-based congestion control may react more rapidly than queue-based scheme, Congestion Control and Fairness for Many-to-one Routing in Sensor Networks [3] is another rate assignment scheme that uses a different congestion detection mechanism than IFRC. Data gather tree algorithm [4] is researched to eliminate the congestion in WSNs.

The classification of the congestion in WSNs includes node-congestion and link-congestion [5]. (1) nodecongestion: the traffic need to send is more than the ability of the sensor node, which causes the buffer overflow, packet loss or queue delay increasing. (2) link-congestion: several adjacent nodes compete one shared channel at the same time, which will generate an access conflict, increase packet service time or reduce the link utilization and network throughput. Based on the discussion above, it is obvious that the congesion control in WSNs should consider both the queue length and the traffic rate. Sliding mode control [9] solves both node-congestion and linkcongestion in WSNs, but whose performace deteriorates in time-variable network conditions. The time-variable network parameter and topology in WSNs is considered as the syetem noise which can be suppress by Generalized minimum variance(GMV) [6] effectively. In conclusion, the generalized minimum variance is deployed in the congestion control for WSNs.

The rest of the paper is structured as follows: section 2 presents the congestion model based on the fluid-flow theory, small signal linearization and the bilinear Ztransform for WSNs. In section 3, congestion control algorithm based on Generalized minimum variance(GMVCC) is provided. Section 4 contains a performance evaluation of the proposed algorithm and a 
comparison with Sliding mode control[9] by Ns-2. The conclusion of this paper is presented in Section 5.

\section{MODEL ANALYSIS}

The fluid-flow model [7] describes a long-lived TCP connection with an additive increase and multiplicative decrease (AIMD) strategy and is given by the following nonlinear differential equations:

$$
\begin{aligned}
& W(t)=\frac{1}{R(t)}-\frac{W(t)}{2} \frac{W(t-R(t))}{R(t-R(t))} p(t-R(t)) \\
& \&(t)=-C(t)+\frac{N(t)}{R(t)} W(t)
\end{aligned}
$$

where $W$ is the average congestion window size(packets), $q$ is the average queue length(packets), $R(t)$ is the round trip time(secs), and $p$ is the probability of packet mark/drop in AQM. $C, T_{p}$ and $N$ denote the link capacity(packets/sec), the propagation delay(secs), and the connection number, respectively. $1 / R(t)$ indicates the additive increase strategy and $W(t) / 2$ means the multiplicative decrease strategy. Simulation results demonstrated that this model accurately captured the dynamics of TCP.

Employing small signal linearization with the equilibrium point $\left(W_{0}, q_{0}, p_{0}\right)$, the simplified dynamics is given as

$$
\begin{aligned}
\dot{x_{1}}(t) & =-\frac{N_{0}}{R_{0}^{2} C_{0}}\left(x_{1}(t)+x_{1}\left(t-R_{0}\right)\right)-\frac{R_{0} C_{0}^{2}}{2 N_{0}^{2}} u\left(t-R_{0}\right) \\
& -\frac{1}{R_{0}^{2} C_{0}}\left(x_{2}(t)-x_{2}\left(t-R_{0}\right)\right), \\
\dot{x}_{2}(t) & =\frac{N_{0}}{R_{0}} x_{1}(t)-\frac{1}{R_{0}} x_{2}(t),
\end{aligned}
$$

Where $x_{1}(t)=\delta W=W(t)-W_{0}, x_{2}(t)=\delta q=q(t)-q_{0}$, $u(t)=\delta p=p(t)-p_{0}$. The equilibrium point $\left(W_{0}, q_{0}\right.$, $p_{0}$ ) is defined by $W^{\&}=0$ and $\&=0$, so that

$$
\begin{aligned}
& W_{0}=\sqrt{\frac{2}{p_{0}}}=\frac{R_{0} C_{0}}{N_{0}}=\frac{q_{0}+C_{0} T_{p}}{N_{0}}, \\
& p_{0}=\frac{2 N_{0}^{2}}{\left(R_{0} C_{0}\right)^{2}}=\frac{2 N_{0}^{2}}{\left(q_{0}+C_{0} T_{p}\right)^{2}}, \\
& R_{0}=\frac{q_{0}}{C_{0}}+T_{p} .
\end{aligned}
$$

The outgoing traffic for sensor node $\mathrm{i}$ is defined as $x_{i}=x_{1} / R_{0}$, then the model (3) for sensor node $\mathrm{i}$ is rewritten as

$$
\dot{\delta q_{i}}(t)=N_{0} x_{i}(t)-\frac{1}{R_{0}} \delta q(t)
$$

Considerring both the link-congestion and the nodecongestion, the wireless sensor network model [8] is proposed in order to estimate the outgoing traffic for sensor node $\mathrm{i}$.

$$
d x_{i}=\left(u_{i}-x_{i}\right) x_{i} \frac{\sum x_{i}-\frac{q_{0}-q(t)}{T}-C}{\sum x_{i}} d t
$$

where $x_{i}$ is the outgoing traffic and $u_{i}$ is the input traffic. The linear equation[9] based on the equilibrium point $\left(u_{0}\right.$, $\left.x_{0}, q_{d}\right)$ is given as

$$
\underset{i}{\&}(t)=\mathbf{A} x_{i}(t)+\mathbf{B} u_{i}(t)
$$

where

$$
\begin{aligned}
\mathbf{A}= & \left(\sum x_{j}\right)^{-2}\left(u_{0}-2 x_{0}\right)\left(\sum x_{j}-\frac{q_{0}-q_{d}}{R_{0}}-C_{0}\right) \\
& +\left(u_{0}-x_{0}\right) x_{0}\left(4-\frac{q_{0}-q_{d}}{R_{0}}-C_{0}\right) \\
B & =x 0 \frac{\sum x_{j}-\frac{q_{0}-q_{d}}{R_{0}}-C_{0}}{\sum x_{j}}, \quad q_{0}=\frac{C_{0}}{N_{0}}
\end{aligned}
$$

Based on the Laplace transformation of the model (4) and (6), the wireless sensor network model can simplified to a single input single output system, as shown in Fig .1, where

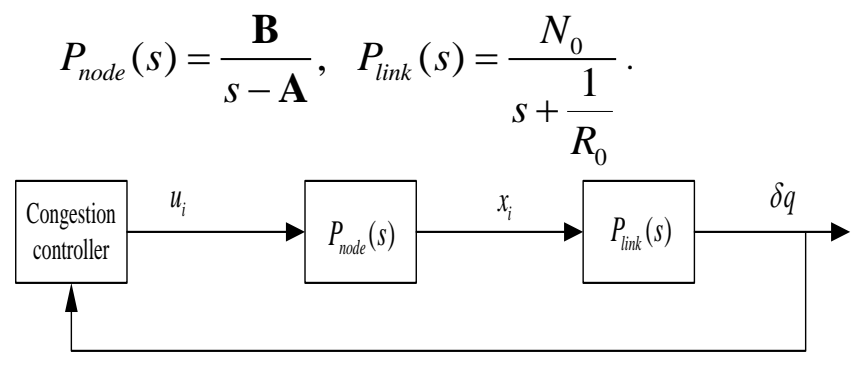

Figure 1. Congestion control system

The wireless sensor network model can be described by using transfer function:

$$
G(s)=\frac{\mathbf{B} N_{0}}{(s-\mathbf{A})\left(s+\frac{1}{R_{0}}\right)}
$$

Using bilinear Z-transform with $T=R_{0}$, we can get the discrete transfer function,

$$
\begin{aligned}
G(z) & =\left.G(s)\right|_{s=\frac{2}{T} \frac{Z-1}{Z+1}} \\
& =\frac{B N_{0} R_{0}^{2}(z+1)^{2}}{3\left(2-A R_{0}\right) z^{2}-\left(8+2 A R_{0}\right) Z+\left(2+A R_{0}\right)}
\end{aligned}
$$

\section{GENERALIZED MINIMUM VARIANCE CONTROLLER}

The GMV approach is a suitable control method, especially under measurement noise. The time-variable network parameter and topology in WSNs is considered as the syetem noise. So the GMV controller [10] is introduced in this section firstly.

Assuming $u(k)$ is the input of the system, $y(k)$ is the output and $e(k)$ is the system noise, then the system equation can be written as: 


$$
A\left(Z^{-1}\right) y(k)=Z^{-d} B\left(Z^{-1}\right) u(k)+e(k)
$$

where $d$ is the system delay, $A\left(Z^{-1}\right)$ is a single polynomial of order $\mathrm{n}$ with coefficients $a_{i}$, and $B\left(Z^{-1}\right)$ is a general polynomial of order $\mathrm{m}$ with coefficients $b_{i}$.

The reason why designing the generalized minimum variance controller is to minimize the following performance index,

$$
J_{G M V}=E\left\{\left(P\left(Z^{-1}\right) y(k+d)-R_{0} y_{r}(k)+q_{0} u(k)\right)\right\}
$$

where $y, u$ and $y_{r}$ are the output, control signal, and input reference signals, respectively. $R_{0}, q_{0}$, and $P\left(Z^{-1}\right)$ are the design parameters determined by the user. Through minimizing the variance of the output, GMV controller has been designed to reduce the noise effects. While designing the controller for a minimum phase system, the following Diophantine equation is required to solve,

$$
A\left(Z^{-1}\right) E\left(Z^{-1}\right)+Z^{-d} F\left(Z^{-1}\right)=1
$$

where

$$
\begin{aligned}
& E\left(Z^{-1}\right)=e_{0}+e_{1} Z^{-2}+\cdots+e_{d-1} Z^{-(d-1)} \\
& F\left(Z^{-1}\right)=f_{0}+f_{1} Z^{-2}+\cdots+f_{d-1} Z^{-(n-1)}
\end{aligned}
$$

According to the closed loop transfer function of this controller, the control signal can be obtained:

$$
u(k)=\frac{R_{0} y_{r}(k)-F\left(Z^{-1}\right) y(k)}{q_{0}+E\left(Z^{-1}\right) B\left(Z^{-1}\right)}
$$

The following lemma provides a necessary and sufficient condition for the stability of this GMV-based controller.

Lemma 1 [11]. The necessary and sufficient condition for the closedloop system to be stable under the GMV control is that all the roots ofthe polynomial $G_{d}\left(z^{-1}\right)=A\left(z^{-1}\right) Q\left(z^{-1}\right)+B\left(z^{-1}\right) C\left(z^{-1}\right)$ belong to the open unit disk, and the polynomial pairs $(\mathrm{Q}, \mathrm{C}),(\mathrm{A}, \mathrm{C})$, and $(\mathrm{B}, \mathrm{Q})$ have no common zeros outside the unit disk.

\section{Simulation}

In this section, we verify the proposed GMVCC via simulation using the Ns-2 simulator. The wireless sensor network topology is addressed as shown in Fig .2, which is the same with reference [9]. The following numerical values are considered as the system parameters: $\mathrm{N}_{0}=144$, $\mathrm{C}_{0}=20160$ packet $/ \mathrm{s}, \mathrm{q}_{0}=140$ packet, $\mathrm{p}_{\mathrm{d}}=120$ packet, $\mathrm{u}_{0}=25$, $\mathrm{R}_{0}=1 \mathrm{~s}$.

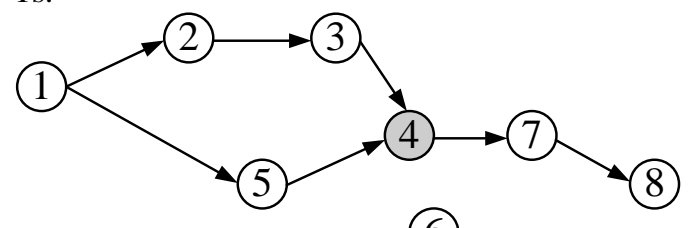

(6)

Figure 2. Network topology

The polynomials for the GMV controller method in Section 3 can be written as:

$$
\begin{aligned}
& F\left(z^{-2}\right)=f_{0}+f_{1} z^{-1}=2.1-1.05 z^{-1} \\
& E\left(z^{-1}\right)=e_{0}=1
\end{aligned}
$$

\section{A. Experiment 1}

Now we look at the bottleneck sensor node 4 running GMVCC. In order to verify the robustness of the proposed GMVCC, the network condition is changed as follow: (a) initial condition; (b) round trip time $R_{0}$ is converted from $1 \mathrm{~s}$ to $1.2 \mathrm{~s}$; (c) 2 new sensor nodes add in the wireless sensor network at 80 s randomly; (d) 2 existing sensor nodes remove at $80 \mathrm{~s}$. Fig .3 depictes the instantaneous queue length of the bottleneck router in variable network condition. As shown in Fig .3, the instantaneous queue length maintains round the equilibrium value. The simulation results show that GMVCC predicts the utilization of the link efficiently, provides early feedback for congestion and has a good robustness when network condition changes or modeling is uncertain.

\section{B. Experiment 2}

In this simulation experiment, we compare the performance of our GMVCC and sliding mode variable structure controller [9]. Changing the packet generating rate, Fig .4 shows the packet drtp ratio and the throughput between the two congestion control strategies. The simulation results show that the proposed GMVCC provides better network performance and higher quality of service in the time-variable network conditon. As discussed in section 1, the energy usage is the key factor in WSN. The lost parameter $\eta$ is used to measure the energy efficiency of the whole network, which is defined in [8] The lost parameter $\eta$ at difference packet generating rate is shown in table 1.

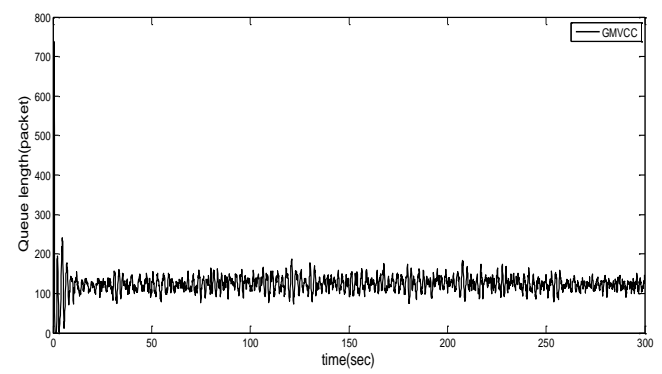

(a)

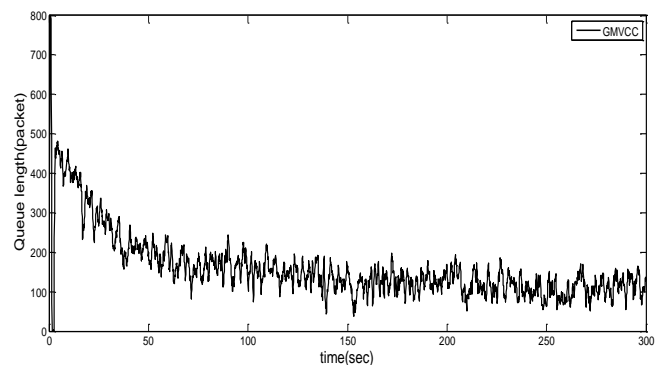

(b)

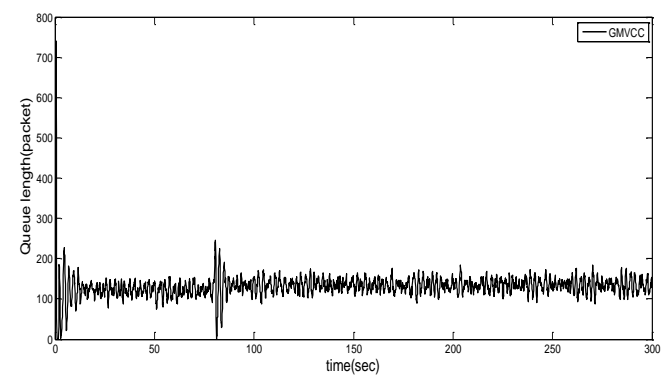

(c) 


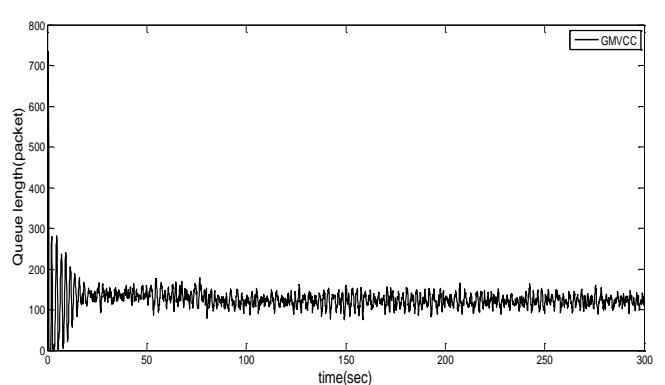

(d)

Figure 3. Queue length for GMVCC

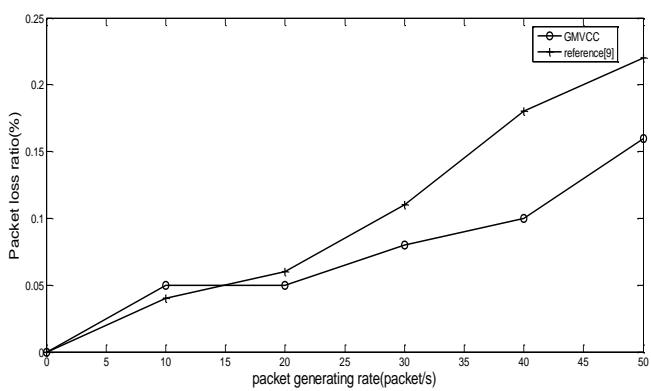

(a) packet drtp ratio

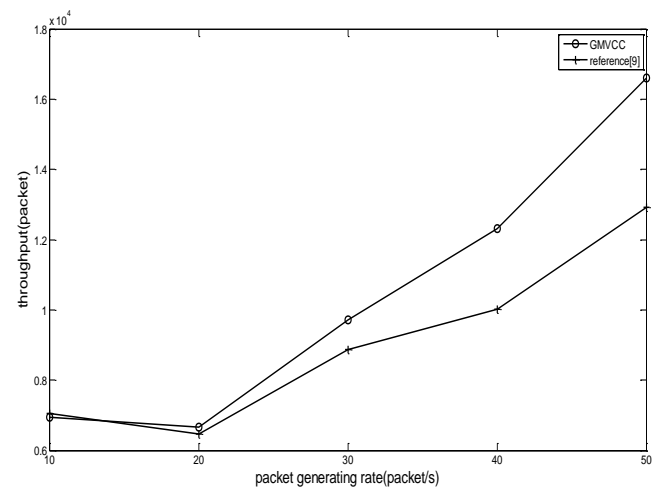

(b) throughput

Figure 4. Network performance

TABLE I. LOSS PARAMETER H AT DIFFERENCE PACKET GENERATING RATE

\begin{tabular}{|c|c|c|c|c|c|}
\hline $\begin{array}{c}\text { packet generating } \\
\text { rate (packet/s) }\end{array}$ & 10 & 20 & 30 & 40 & 50 \\
\hline GMVCC & 0.233 & 0.311 & 0.547 & 1.034 & 1.874 \\
\hline Reference[9] & 0.198 & 0.302 & 0.487 & 0.842 & 1.535 \\
\hline
\end{tabular}

\section{CONCLUSION}

The congestion problem is unavoidable because of the many-to-one characteristic in the wireless sensor network, which leads to packets drops at the buffers, increased delays, wasted energy and requires retransmissions. Based on the generalized minimum variance controller, a novel congestion control algorithm (GMVCC) is introduced in this paper, which improves the quality of service in WSNs. In the same time, the performance of GMVCC is not so perfect in time-variable condition, especially within the variable round trip time. Therefore, in order to fill this gap adaptive control combined with GMV is our further research.

\section{ACKNOWLEDGMENT}

The author is indebted to the the National Natural Science Foundation of China(61203048, 61201212, 61304047), the Natural Science Foundation of Jiangsu Province of China(BK2011376) for financial support.te.

\section{REFERENCES}

[1] Pavlos Antoniou, Andreas Pitsillides, Andries Engelbrecht, et al. Congestion control in wireless sensor networks based on bird flocking behavior. Computer Networks. 2013, 57(5):1167-1191.

[2] S. Rangwala, R. Gummadi, R. Govindan, K. Psounis. Interferenceaware fair rate control in wireless sensor networks, in: Proceedings of ACM SIGCOMM Symposium on Network Architecture and Protocols, 2006

[3] C.T. Ee, R. Bajcsy, Congestion control and fairness for many-toone routing in sensor networks, in: Sensys 2004

[4] Shi W R,Tang Y J,Wang Y X.Data gather tree algorithm based on congestion control in wireless sensor networks. Acta Automatica Sinica,2010,36(8):823-828.

[5] Sun L M, Li B, Zhou X Y. A survey of congestion control technology for wireless sensor networks. Journal of Computer Research and Development. 2008, 45(1): 63-72.

[6] R. Barzamini, M. Shafiee, A. Dadlani. Adaptive generalized minimum variance congestion controller for dynamic TCP/AQM networks. Computer Communications. 2012, 35(2):170-178.

[7] Misra V, Gong W B, Towsley D. Fluid-based analysis of a network of AQM routers supporting TCP flows with an application to RED. Proceedings of the ACM SIGCOMM'00. 2000: 151-160.

[8] Ouyang Ying, Lin Chuang, Ren Fengyuan, et al. Design and analysis of a backpressue congestion control algorithm in wireless sensor network. The $8^{\text {th }}$ Int Conf on Parallel and Distributed Computing, Aplications and Technologies. Adelaide: University of Adelaide, 2007: 43-420.

[9] LI Mingwei, JING Yuanwei, CHEN Xiangyong. Cross-layer congestion control for wireless sensor network based on sliding mode variable structure. Control and Decision. 2012, 27(3): 451454.

[10] K. J. Astrom, B. Wittenmark, Adaptive Control, second ed., Addison-Wesley, Reading, MA, 1995.

[11] P. Ioannou, B. Fidan, Adaptive Control Tutorial, Cambridge University Press, 2006. 